\title{
Epidemiology of urticaria including physical urticaria and angioedema in Korea
}

\author{
Jeong-Hun $\mathrm{Seo}^{1}$ and Jae-Woo Kwon ${ }^{1,2}$
}

\begin{abstract}
${ }^{1}$ Department of Internal Medicine, Kangwon National University Hospital, Chuncheon; ${ }^{2}$ Department of Allergy and Clinical Immunology, Kangwon National University School of Medicine, Chuncheon, Korea
\end{abstract}

Received: June 6, 2017

Revised : July 9, 2017

Accepted: December 4, 2017

\author{
Correspondence to \\ Jae-Woo Kwon, M.D. \\ Department of Internal Med- \\ icine, Kangwon National Uni- \\ versity Hospital, 156 Baengnyeo- \\ ng-ro, Chuncheon 24289, Korea \\ Tel: +82-33-258-9370 \\ Fax: +82-33-258-2404 \\ E-mail: legent@hanmail.net
}

Background/Aims: Despite the high burden and frequency of urticaria, its epidemiology is not well known. We investigated the epidemiology of various type of urticaria in Korea and changes in its annual prevalence over 5 years.

Methods: We used data from the 2010 to 2014 Health Insurance Review and Assessment database, which covers the claims of $97.0 \%$ of the South Korean population. Patients aged $>10$ years old were included in this study. The presence of urticaria was identified on physician-certified diagnoses using the International Classification of Diseases 1oth Revision (ICD-10) codes that include various type of urticaria (L500-L509) and angioedema ( $\mathrm{T}_{7} 83$ ). Epidemiology of all type of urticaria, physical urticaria and angioedema were investigated.

Results: The prevalence of all-type urticaria over the 5 years was $4.5 \%$ with a peak in individuals, especially females, aged 30 to 59 years. The age-specific prevalence of all-type urticaria increased with age from the 10- to 19-year age group to the 70- to 79-year age group. The prevalence of dermographism, cholinergic urticaria, and angioedema were $0.12 \%, 0.025 \%$, and $0.027 \%$, respectively. Cholinergic urticaria was most prevalent in those aged 10 to 29 years with male predominance. The annual prevalence of all-type urticaria, dermographism, and angioedema increased over the 5 years.

Conclusions: The prevalence of urticaria has increased annually in Korea. Cholinergic urticaria showed unique distribution in its age and gender, and angioedema showed remarkable increases in annual prevalence, although the prevalence estimation is still exploratory and diagnosis of urticaria based on ICD-10 codes need to be validated.

Keywords: Urticaria; Angioedema; Epidemiology; Prevalence; Republic of Korea

\section{INTRODUCTION}

Urticaria, first described by Hippocrates, is a common reason for doctor and emergency department visits [1]. It has been estimated that approximately $20 \%$ of the population will experience at least one episode of urticaria during their lifetime $[1,2]$. Urticaria is a heterogeneous group of diseases defined by pruritic wheal and flare-type skin reactions, with or without angioedema, that usually persist for less than 24 hours [2]. Urticaria is also a disease with high burden. Among allergic diseases, patients with urticaria or angioedema showed higher emergency room usage than did patients with other allergy diseases [3]. Especially patients with chronic urticaria, characterized by urticarial episodes lasting longer than 6 weeks, reported a markedly reduced quality of life and high direct medical expenses in previous studies $[4,5]$. The majority of patients with chronic urti- 
caria have urticarial lesions daily and report emotional distress similar to that experienced by patients with ischemic heart disease [4]. Physical urticaria makes up a subset of chronic urticaria and is responsible for approximately $20 \%$ to $30 \%$ of cases of chronic urticaria [6]. Physical urticaria is induced by a wide variety of environmental stimuli, such as exercise, temperature changes, cold, heat, pressure, and sunlight.

Although urticaria is one of the most frequent diseases resulting in consultations and it causes a high burden characterized by a reduction in quality of life and performance $[3,7]$, few studies have investigated the prevalence of this condition and there are little data concerning changes in its prevalence [1-3,7]. Especially for physical urticaria, although simple physical urticaria is known to be relatively common, prevalence data about symptomatic physical urticaria have been rarely established and there is no data concerning changes in its prevalence [6].

This study estimated the prevalences and changes in the prevalences of various type of urticaria and angioedema over 5 years using the National Health Insurance (NHI) claims database in Korea. The NHI program covers the claims of $97.0 \%$ of the Korean population, which accounts for approximately 50 million individuals. Data were about clinical urticaria or angioedema diagnosed by clinicians.

\section{METHODS}

\section{Study population and data sources}

We used the 2010 to 2014 Health Insurance Review and Assessment Service (HIRA) database; HIRA is the government agency that supervises all medical services in Korea [8]. The NHI program in Korea was initiated in 1977 and achieved universal coverage of the population by 1989 . The HIRA service covers the claims of $97.0 \%$ of the Korean population ( -50 million individuals); the remaining $3 \%$ of the population is covered by the Medical Aid Program. The Korean population is composed almost completely of ethnic Koreans. Disease statistics from HIRA were collected based on medical care expense details from hospitals and pharmacies. We used the online open database provided by HIRA since 2010 (access at http://opendata.hira.or.kr/op/opc/olap4thDsInfo. do), which shows the annual number of inpatients and outpatients with specific diseases from 2010 to 2014 according to age group (divided into 10-year-old), sex, and classification of hospital (tertiary hospital). Diagnosis of diseases is based on main disease (International Classification of Diseases [ICD] code). When a single patient was diagnosed with several diseases, the physician selected the main disease based on treatment burden. It is impossible to know whether the patients have multiple types of urticaria or not. And multiple visits with same diagnosis of one patient during same year were counted as one case of that diagnosis during that year.

Identification of the presence of urticaria as the main disease was based on physician-certified diagnoses using ICD-10 codes, which included allergic urticaria (L500), idiopathic urticaria ( $\left.\mathrm{L}_{501}\right)$, cold and heat urticaria $\left(\mathrm{L}_{502}\right)$, dermographism (L503), vibratory urticaria (L504), cholinergic urticaria (L505), contact urticaria (L506), chronic or recurrent urticaria (L508), unspecified urticaria (L509), and angioedema ( $\left.\mathrm{T}_{7} 83\right)$. In current study, 'clinical urticaria' is defined as urticaria which caused patients to visit hospital and diagnosed as main problem by clinician. All-type urticaria was defined to include all of the aforementioned urticaria types. Patients aged $>10$ years old were included in study population because o to 10 age group include infants and young children for whom the diagnosis of physical urticaria and angioedema could be difficult in clinical field.

We estimated the prevalences of angioedema $\left(\mathrm{T}_{7} 83\right)$ and physical urticaria (L502, L503, L505) which have relatively clear phenotypes, chronic or recurrent urticaria (L508) and all-type urticaria. The annual prevalence of urticaria was calculated by dividing the total number of patients with urticaria by the number of residents registered in Korea that year according to the National Statistics Office. The age-specific prevalence was calculated by dividing the number of urticaria patients in a specific age group by the number of registered residents in that age group in Korea. This study received the Institutional Review Board approval of Kangwon National University Hospital (IRB File No. KNUH-2018-04-003-001).

\section{Statistical analysis}

The prevalences of various types of urticaria were estimated according to the year and the age and sex of patients. Changes in the trends in the annual prevalence of urticaria over the 5 years were assessed using the Co- 
chran-Armitage trend test. A p value of less than 0.05 was considered to indicate statistical significance. All statistical analyses were performed using the SAS software version 9.4 (SAS Institute, Cary, NC, USA).

A
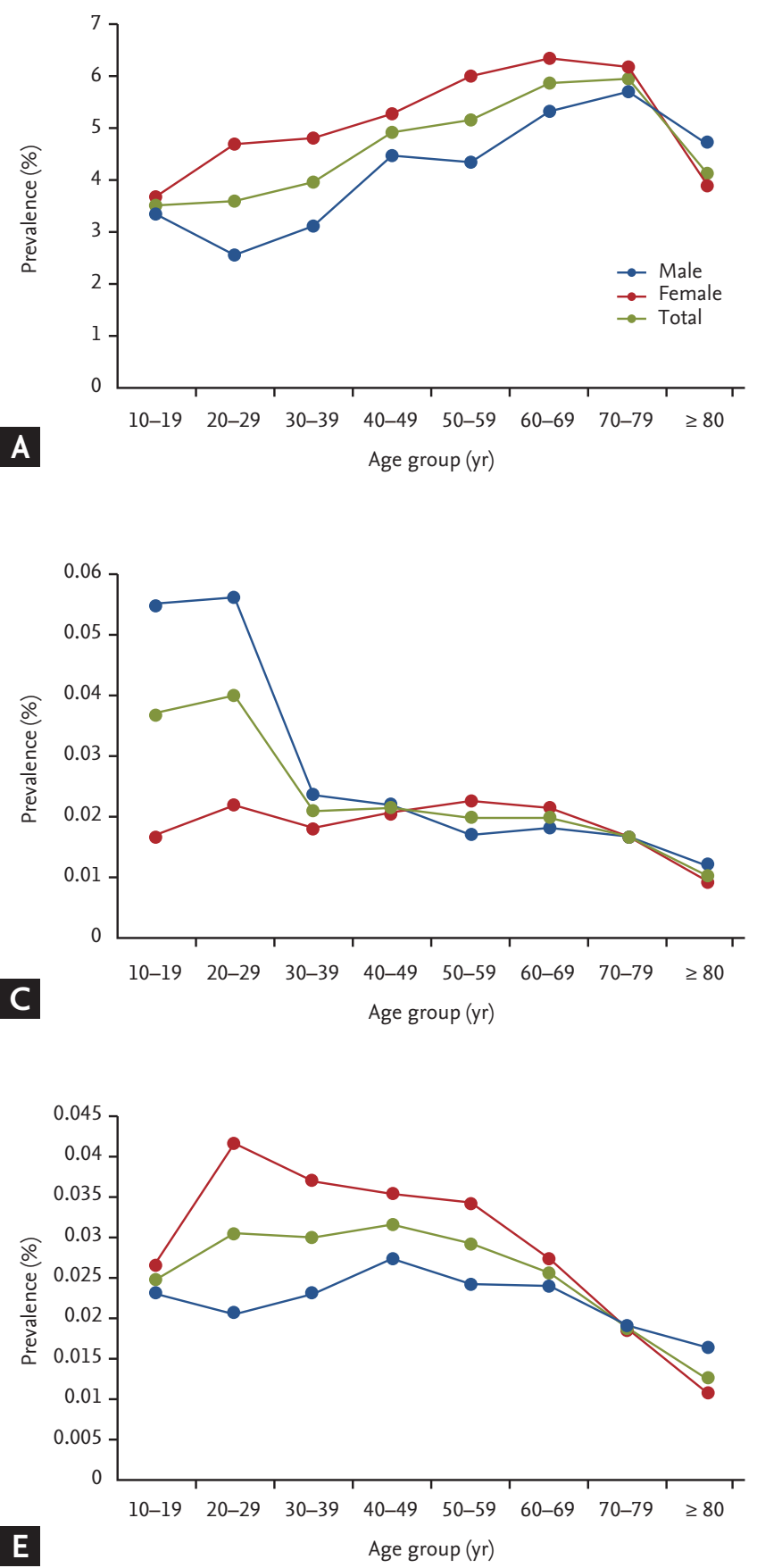

\section{RESULTS}

\section{All-type urticaria}

The prevalence of all-type urticaria among population
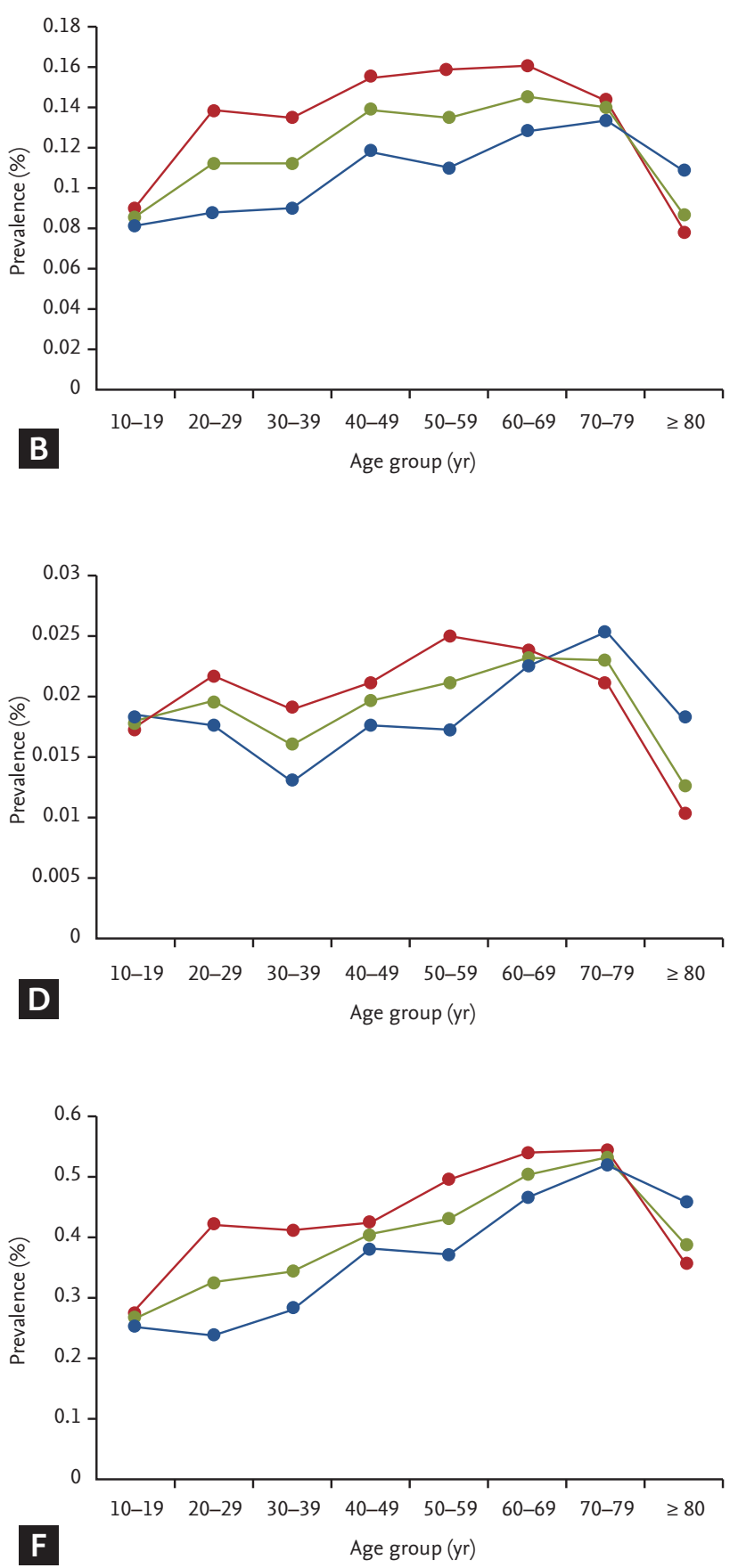

Figure 1. Age-specific prevalence of urticaria in Korea. The numbers represent the percentages of gender distribution among patients in each age group. Blue line presents age-specific prevalence of male patients, red line presents age-specific prevalence of female patients, and dot line presents age-specific prevalence of total patients. (A) All-type urticaria, (B) dermographism, (C) cholinergic urticaria, (D) cold/heat urticaria, (E) angioedema, and (F) chronic or recurrent urticaria. 


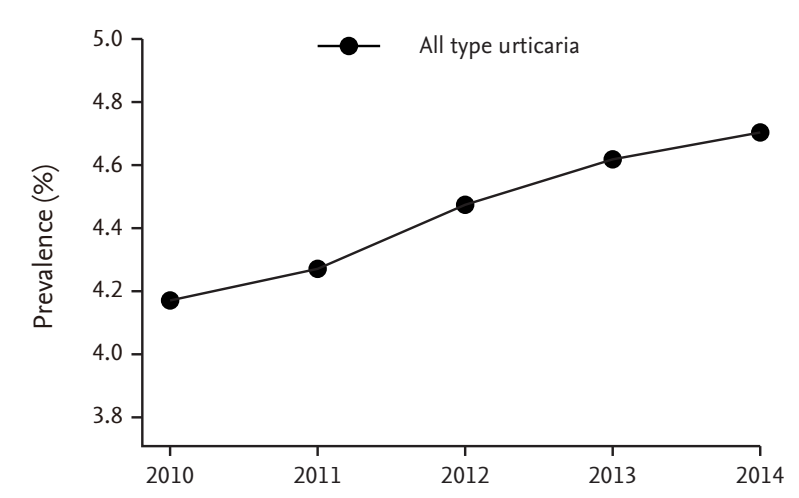

$\mathbf{A}$

Year

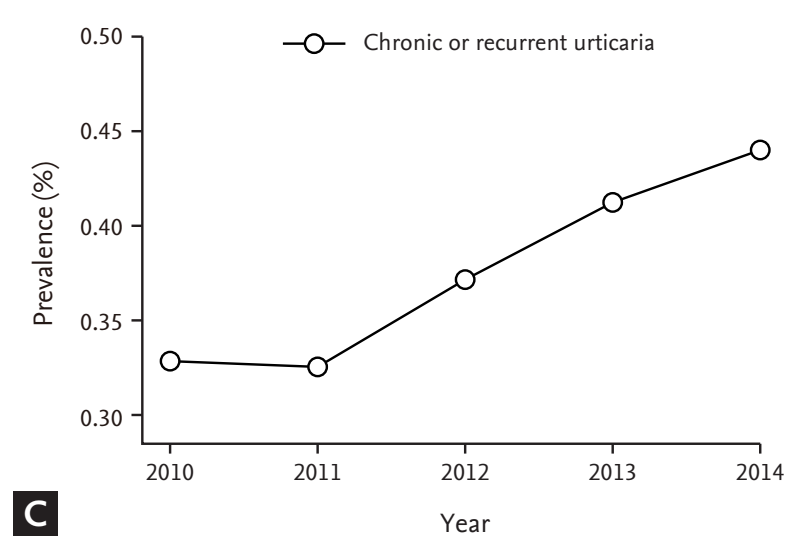

$\geq 10$ years old over 5 years was $4.5 \%$ (Table 1 ) with a female predominance $(58 \%)$ and a peak in the distribution in the 30- to 59-year age group (Table 2). However, the age-specific prevalence of all-type urticaria over 5 years was higher in older individuals, and it increased gradually from the 10- to 19-year age group to the 70 to 79-year age group (Fig. 1A). The annual prevalence of all-type urticaria increased from $4.2 \%$ in 2010 to $4.7 \%$ in 2014 (p<0.0001) (Fig. 2).

\section{Dermographism}

The prevalence of dermographism over 5 years was $0.12 \%$ and 1.4-fold higher in females than in males among patients aged $\geq 20$ years (59\% vs. 41\%). Dermographism was most prevalent in patients aged 40 to 59 years (Table 2), and the age-specific prevalence of dermographism was highest among those aged 40 to 79 years (Fig. 1B). Moreover, the annual prevalence of dermographism gradually increased from $0.114 \%$ to $0.122 \%$ over the 5 years $(p<$ 0.0001 ) (Fig. 2B).

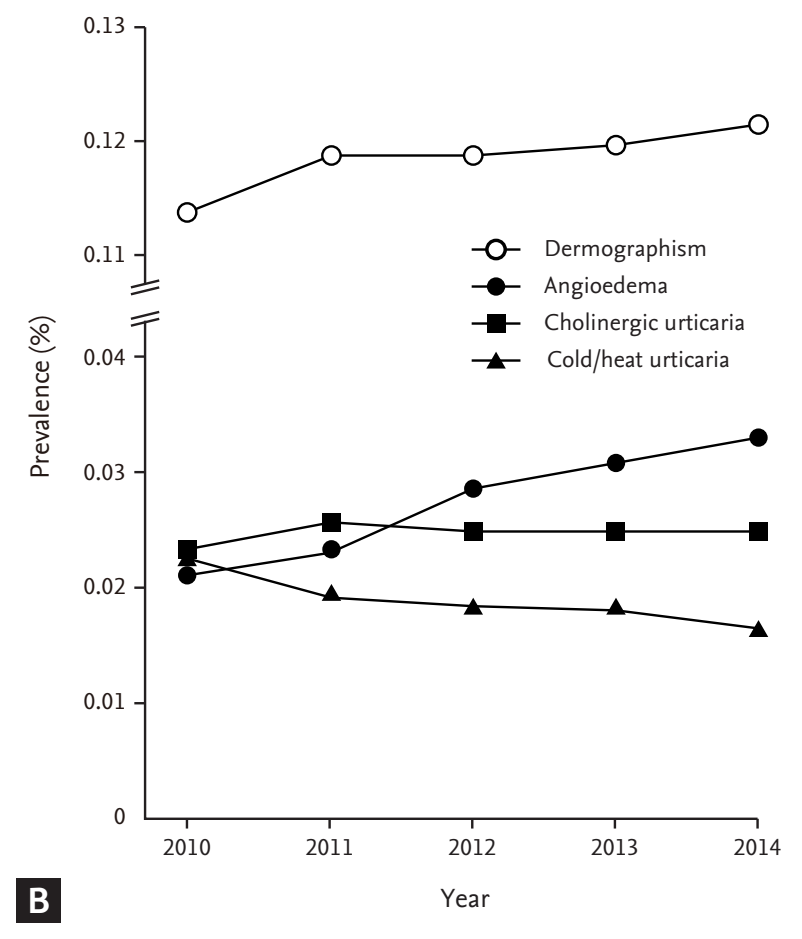

Figure 2. Changes in annual prevalence of various types of urticaria over 5 years in Korea. (A) All-type urticaria, (B) dermographism, cholinergic urticaria, cold/heat urticaria, and, angioedema, and (C) chronic or recurrent urticaria.

\section{Cholinergic urticaria}

The prevalence of cholinergic urticaria over 5 years was $0.025 \%$, and it was 2.5 -fold higher in male than female patients 10 to 39 years old ( $71 \%$ vs. $29 \%$ ). On the other hand, there were fewer male than female patients with this condition among those $\geq 40$ years old ( $44 \%$ vs. $56 \%$ ). Cholinergic urticaria was most prevalent in the 10- to 29-year age group (Table 2), and its prevalence decreased gradually with increasing age (Fig. $1 \mathrm{C}$ ). There were no significant changes in the annual prevalence of cholinergic urticaria from 2011 to 2014 (Fig. 2B).

\section{Cold and heat urticaria}

The prevalence of cold and heat urticaria over 5 years was $0.019 \%$, and it was 1.2-fold higher in female than in male patients ( $55 \%$ vs. $45 \%$ ). Cold and heat urticaria was most prevalent in patients aged 40 to 59 years (Table 2), whereas the age-specific prevalence was highest in the 60- to 79-year age group (Fig. 1D). The annual prevalence of cold and heat urticaria gradually decreased from 
Table 1. Prevalence of various types of urticaria over 5 years in Korea

\begin{tabular}{lc}
\hline Type of urticaria & Prevalence, \% \\
\hline All-type urticaria $^{\mathrm{a}}$ & 4.544 \\
Dermographism & 0.121 \\
Cholinergic urticaria & 0.023 \\
Cold/heat urticaria & 0.019 \\
\hline Angioedema & 0.028 \\
Chronic or recurrent urticaria & 0.385 \\
\hline
\end{tabular}

${ }^{\text {aAll-type urticaria include the above-mentioned physical }}$ urticaria, angioedema, chronic, or recurrent urticaria, and other types of urticaria diagnosis (International Classification of Diseases [ICD] code) including allergic urticaria (L500), idiopathic urticaria (L501), vibratory urticaria (L504), contact urticaria (L506), and unspecified urticaria (L509).
$0.023 \%$ in 2010 to $0.016 \%$ in 2014 (p< $<0.0001$ ) (Fig. $2 \mathrm{~B})$.

\section{Angioedema}

The prevalence of angioedema over 5 years was $0.027 \%$. A higher proportion of females than males ( $59 \%$ vs. $41 \%$ ) was affected, and patients in the 30- to 49-year age group were most commonly affected (Table 2). The age-specific prevalence was high in patients aged 20 to 49 , and a subsequent gradual decrease with age (Fig. $1 \mathrm{E}$ ). The annual prevalence of angioedema increased from $0.021 \%$ to $0.033 \%$ over the 5 years $(p<0.0001)$ (Fig. $2 \mathrm{~B})$.

\section{Chronic or recurrent urticaria}

The prevalence of chronic or recurrent urticaria over 5 years was $0.38 \%$. There were 1.4 -fold more females than males among patients with this condition who were

Table 2. Age and sex distribution of patients with urticaria in Korea

\begin{tabular}{|c|c|c|c|c|c|c|c|c|c|}
\hline \multirow{2}{*}{ Type of urticaria } & \multirow{2}{*}{ Total, \% } & \multicolumn{8}{|c|}{ Age group in years, $\%$} \\
\hline & & $10-19$ & $20-29$ & $30-39$ & $40-49$ & $50-59$ & $60-69$ & $70-79$ & $\geq 80$ \\
\hline \multicolumn{10}{|c|}{ All-type urticaria $(\mathrm{n}=2,060,971)$} \\
\hline Total & & 11 & 11.7 & 15.6 & 19 & 19.4 & 12.5 & 8.49 & 2.25 \\
\hline Female & 58 & 50 & 62 & 60 & 58 & 58 & 56 & 60 & 66 \\
\hline Male & 42 & 50 & 38 & 40 & 42 & 42 & 44 & 40 & 34 \\
\hline \multicolumn{10}{|c|}{ Dermographism $(\mathrm{n}=54,862)$} \\
\hline Total & & 10 & 13.6 & 16.6 & 20.1 & 18.9 & 11.6 & $7 \cdot 42$ & 1.76 \\
\hline Female & 58 & 50 & 59 & 59 & 61 & 59 & 57 & 60 & 63 \\
\hline Male & 42 & 50 & 41 & 41 & 39 & 41 & 43 & 40 & 37 \\
\hline \multicolumn{10}{|c|}{ Cholinergic urticaria $(\mathrm{n}=11,429)$} \\
\hline Total & & 20.9 & $23 \cdot 3$ & 14.8 & 14.8 & 13.2 & $7 \cdot 6$ & 4.23 & 0.99 \\
\hline Female & 40 & 22 & 26 & 43 & 54 & 57 & 56 & 58 & 65 \\
\hline Male & 60 & 78 & 74 & 57 & 46 & 43 & 44 & 42 & 35 \\
\hline \multicolumn{10}{|c|}{ Cold/heat urticaria $(\mathrm{n}=8,762)$} \\
\hline Total & & 13.2 & $14 \cdot 9$ & 14.7 & $17 \cdot 7$ & 18.6 & 11.6 & 7.64 & 1.59 \\
\hline Female & 55 & 46 & 53 & 58 & 59 & 59 & 53 & 54 & 58 \\
\hline Male & 45 & 54 & 47 & 42 & 41 & 41 & 47 & 46 & 42 \\
\hline \multicolumn{10}{|c|}{ Angioedema $(\mathrm{n}=12,669)$} \\
\hline Total & & 12.6 & 16.2 & 19.2 & 19.9 & 17.8 & 8.86 & $4 \cdot 33$ & 1.1 \\
\hline Female & 59 & 51 & 65 & 60 & 61 & 58 & 55 & 58 & 61 \\
\hline Male & 41 & 49 & 35 & 40 & 39 & 42 & 45 & 42 & 39 \\
\hline \multicolumn{10}{|c|}{ Chronic or recurrent urticaria $(\mathrm{n}=174,579)$} \\
\hline Total & & $9 \cdot 77$ & 12.5 & 16 & 18.5 & 19.1 & 12.6 & 8.91 & 2.48 \\
\hline Female & 57 & 50 & 62 & 58 & 57 & 57 & 55 & 59 & 65 \\
\hline Male & 43 & 50 & 38 & 42 & 43 & 43 & 45 & 41 & 35 \\
\hline
\end{tabular}


aged $\geq 20$ years ( $58 \%$ vs. $42 \%$ ). Chronic or recurrent urticaria was most prevalent in the 40 - to 59 -year age groups (Table 2). The age-specific prevalence over 5 years was lowest in the 10- to 19-year age group (0.27\%), and it gradually increased with age to the 70- to 79-year age group (0.53\%) (Fig. 1F). The annual prevalence of chronic or recurrent urticaria increased from $0.33 \%$ to $0.44 \%$ over the 5 years $(p<0.0001$ ) (Fig. $2 \mathrm{C}$ ).

\section{DISCUSSION}

In this study, we estimated the prevalence of all type urticaria, physical urticaria, and angioedema over 5 years using the Korean insurance claim database, which covers the majority of the Korean population. Thus, we were able to estimate the age-specific prevalence of urticaria over 5 years and changes in the annual prevalence of various types of urticaria. Since this is the epidemiologic study about clinical urticaria requiring a hospital visit, comparing our data with those from other studies is difficult, as the majority of previous epidemiological studies concerning urticaria were population-based and relied on questionnaires $[1,7]$.

A recent studies reported that the lifetime prevalence of all types of urticaria was $8.8 \%$ to $10.8 \%$, with a female predominance and a mean age of 35 to 39 years $[3,7]$. The current study showed that, in Korea, the prevalence of all-type urticaria over 5 years was $4.5 \%$ with female predominance; moreover, the annual prevalence of all-type urticaria increased over the 5 years. The prevalence of all-type urticaria was lower than that reported by previous studies, which may be because this study focused on clinical urticaria requiring a hospital visit. Given that the peak distribution of all-type urticaria occurred in the 40- to 59-year age group, which is older than reported previously, and urticaria was more common in older individuals based on the age-specific prevalence, this study suggests that clinical urticaria is important among the older population $[3,7]$. In addition, this study showed that the annual prevalence of all-type urticaria increased over the 5 years. There have been few studies about changes in its prevalence and those studies suggested that the incidence of urticaria had been increased during the past decades $[2,9]$. However, the prevalence of most physical urticaria did not increase. Considering urticaria is one of typical manifestation caused by allergic reaction and most of allergy diseases have increased recently, most of physical urticaria may have different pathophysiology from other non-physical urticaria and allergic hypersensitivity.

Dermographism was the most common type of physical urticaria. In a previous study, dermographism was estimated to occur in approximately $2 \%$ to $5 \%$ of the general population [10], but the symptomatic forms of dermographism are expected to be less common, and no prevalence data have been established for such symptomatic dermographism [6]. This study showed that 1.1\% of the total population of Korea visited hospitals for dermographism each year. Furthermore, the annual prevalence increased over the 5 years. Dermographism, cold and heat urticaria, and angioedema occurred most frequently in female patients in the 30- to 59-year age range, and data on the age-specific prevalence showed a similar pattern of distribution according to age. Several studies using questionnaires have investigated the epidemiology of physical urticaria; however, no studies have been conducted to determine the epidemiology of clinical physical urticaria. Previous studies have reported that physical urticaria accounts for $20 \%$ of cases of chronic urticaria [3,6]; unfortunately, we were unable to identify patients who had both chronic and physical urticaria, which is one of limitations in current study.

Cholinergic urticaria was most prevalent in the 20to 29-year age group, with a male predominance, and showed an opposite sex distribution in patients $\geq 40$ years of age. Several studies based on small samples reported that cholinergic urticaria typically developed during the second or third decade of life and had a male predominance or an equal distribution for both sexes [11-14]. Cholinergic urticaria was associated with different demographic characteristics compared with other types of urticaria, including a higher prevalence among males aged 20 to 29 years of age and a difference in the sex distribution of patients younger and older than 40 years of age. These results suggest that cholinergic urticaria may have a different pathophysiology than other types of physical urticaria.

A total of 29 patients among 100,000 individuals sought medical care for angioedema over 5 years in Korea. Annual prevalence of angioedema also increased over 5 years. Few epidemiological studies have investigated angioede- 
ma. According to a Spanish study, $12.3 \%$ of patients who visited an allergist for urticaria with or without angioedema suffered from isolated angioedema, and 30.8\% suffered from urticaria accompanied by angioedema [3]. Recent studies have reported that increases in the rate of hospital admissions for angioedema and urticaria have been observed in Australia and England [15,16].

It is a major limitation of current study that the prevalence of chronic urticaria was not directly evaluated since patients with chronic urticaria reported a markedly reduced quality of life and high direct medical expenses in previous studies [4,5]. Since there is no specific diagnostic code for chronic urticaria in ICD codes, several studies evaluated the prevalence of chronic urticaria using ICD codes and medication history $[17,18]$. However, we evaluate the ICD-10 code for chronic or recurrent urticaria ( $\left.\mathrm{L}_{5} \mathrm{O} 8\right)$ as a possible indirect indicator for chronic urticaria without reviewing medical records or medication, although it is not validated, and prevalence of chronic or recurrent urticarial over 5 years was $0.38 \%$. Recently, Lee et al. [9] reported the prevalence of chronic urticaria in Korea using same database during same period with current study. They defined chronic urticaria based on ICD code of L50 and medication history; prevalence of chronic urticaria was $2.26 \%$ in Korea and its prevalence tended to increase every year [9]. And they also reported female predominance and higher age-specific prevalence in older population in chronic urticaria. These results were similar with findings of previous studies which reported $0.05 \%$ to 3\% prevalence of chronic urticaria and female prevalence $[1,7,19]$. However, further studies with validated algorithms and medical records are needed about the clear prevalence of chronic urticaria in Korea.

The present study has other limitations to discuss. First, our results were based on the insurance claim data. We estimated the prevalence of urticaria based on clinical urticaria which is defined as urticaria which caused patients to visit hospital and diagnosed as main problem by clinician. So this study did not include cases of patients with urticaria who did not visit a hospital. Thus we may have underestimated the actual prevalence of urticaria in the general population. Second, various types of urticaria were defined only by ICD-10 codes and there are no validations for the coded diagnosis. Although it is diagnosed as main problem by clinicians, the prevalence estimation using ICD-10 codes is still exploratory and diagnosis of urticaria based on ICD-10 codes need to be validated in further studies. This study evaluated several types of physical urticaria and angioedema which have relatively clear phenotype and showed consistent results with previous studies, but diagnostic values of ICD-10 codes for physical urticaria are also still unclear. Third, we were unable to identify patients with more than two types of urticaria simultaneously. Therefore, it is possible that a patient could have been counted twice under different diagnoses if they were diagnosed with two different type of urticaria during different hospital visits.

This study presents the epidemiology of clinical urticaria based on diagnosis (ICD-10 codes) by physicians in real world of Korea. Clinical urticaria is very common, and its prevalence is increasing. Physical urticaria showed different distribution in its age and gender, and different trends in changes of annual prevalence according to various types of physical urticaria. Although the prevalence estimation is still exploratory and diagnosis of urticaria based on ICD-10 codes need to be validated, this epidemiological study may help clinicians to manage and educate patients with urticaria in addition to providing data for future research endeavors.

\section{KEY MESSAGE}

1. Clinical urticaria is very common, and its prevalence is increasing.

2. Various types of physical urticaria showed different distribution in its age and gender, and different trends in changes of annual prevalence.

3. Cholinergic urticaria was most prevalent in those aged 10 to 29 years with male predominance.

\section{Conflict of interest}

No potential conflict of interest relevant to this article was reported.

\section{REFERENCES}

1. Gaig P, Olona M, Munoz Lejarazu D, et al. Epidemiology of urticaria in Spain. J Investig Allergol Clin Immunol 
2004;14:214-220.

2. Maurer M, Weller K, Bindslev-Jensen C, et al. Unmet clinical needs in chronic spontaneous urticaria. A GA2LEN task force report. Allergy 2011;66:317-330.

3. Ferrer M. Epidemiology, healthcare, resources, use and clinical features of different types of urticaria. Alergologica 2005. J Investig Allergol Clin Immunol 2009;19 Suppl 2:21-26.

4. O'Donnell BF, Lawlor F, Simpson J, Morgan M, Greaves MW. The impact of chronic urticaria on the quality of life. Br J Dermatol 1997;136:197-201.

5. Ye YM, Jang GC, Choi SH, et al. KAAACI Work Group report on the management of chronic urticaria. Allergy Asthma Respir Dis 2015:3:3-14.

6. Dice JP. Physical urticaria. Immunol Allergy Clin North Am 2004;24:225-246.

7. Zuberbier T, Balke M, Worm M, Edenharter G, Maurer M. Epidemiology of urticaria: a representative cross-sectional population survey. Clin Exp Dermatol 2010;35:869-873.

8. Lee SY, Jung KY, Lee IK, et al. Prevalence of treated epilepsy in Korea based on National Health Insurance data. J Korean Med Sci 2012;27:285-290.

9. Lee N, Lee JD, Lee HY, Kang DR, Ye YM. Epidemiology of chronic urticaria in Korea using the Korean Health Insurance Database, 2010-2014. Allergy Asthma Immunol Res 2017;9:438-445.

10. Orfan NA, Kolski GB. Physical urticarias. Ann Allergy 1993;71:205-212.
11. Hirschmann JV, Lawlor F, English JS, Louback JB, Winkelmann RK, Greaves MW. Cholinergic urticaria. A clinical and histologic study. Arch Dermatol 1987;123:462-467.

12. Kim JE, Eun YS, Park YM, et al. Clinical characteristics of cholinergic urticaria in Korea. Ann Dermatol 2014;26:189-194.

13. Moore-Robinson M, Warin RP. Some clinical aspects of cholinergic urticaria. Br J Dermatol 1968;80:794-799.

14. Zuberbier T, Althaus C, Chantraine-Hess S, Czarnetzki BM. Prevalence of cholinergic urticaria in young adults. J Am Acad Dermatol 1994;31:978-981.

15. Poulos LM, Waters AM, Correll PK, Loblay RH, Marks GB. Trends in hospitalizations for anaphylaxis, angioedema, and urticaria in Australia, 1993-1994 to 2004-2005. J Allergy Clin Immunol 2007;120:878-884.

16. Gupta R, Sheikh A, Strachan D, Anderson HR. Increasing hospital admissions for systemic allergic disorders in England: analysis of national admissions data. BMJ 2003;327:1142-1143.

17. Cherepanov D, Raimundo K, Chang E, et al. Validation of an ICD-9-based claims algorithm for identifying patients with chronic idiopathic/spontaneous urticaria. Ann Allergy Asthma Immunol 2015;114:393-398.

18. Lapi F, Cassano N, Pegoraro V, et al. Epidemiology of chronic spontaneous urticaria: results from a nationwide, population-based study in Italy. Br J Dermatol 2016; 174:996-1004.

19. Greaves MW. Chronic urticaria. N Engl J Med 1995;332:17671772 . 